\title{
Primary Cutaneous Anaplastic Large Cell Lymphoma: A Rare Case Report in a Young Soldier
}

\author{
Karim $\mathrm{Ml}^{1}$, Ahmed $\mathrm{M}^{2}$, Malakar RK ${ }^{3}$, Nachev R ${ }^{4}$ \\ DOI: https://doi.org/10.3329/jafmc.v17i1.56721
}

\begin{abstract}
Primary cutaneous anaplastic large cell lymphoma (C-ALCL) usually presents with scaly patches or plaques and nodular skin lesion. Most people with C-ALCL have indolent (i.e., chronic, slow growing) lymphomas which are treatable and curable. Histologically cutaneous anaplastic large cell lymphoma is diffuse, non-epidermotropic with anaplastic large lymphoid cells. Immunohistochemically most of the tumor cells express CD30 antigen and prognosis is good. Excision of the lesion followed by radiotherapy and/or low dose methotrexate are the treatment of choice. The present study reports a rare case of 30-year-old-man presenting with multifocal papulonodular lesions in the right forearm and biopsy confirmed that the case is cutaneous anaplastic large-cell lymphoma. He was treated with low dose of methotrexate and patient became completely cured without any residual disease.
\end{abstract}

Key-words: Cutaneous anaplastic large cell lymphoma, Immunohistochemistry, T-cell.

\section{Introduction}

Cutaneous anaplastic large cell lymphoma (C-ALCL) is a type of T cell origin non-Hodgkin lymphoma composed of large cells with an anaplastic, pleomorphic or immunoblastic cytomorphology. The majority (> 75\%) of the tumor cells express the CD30 antigen (WHO 2008)1. This is one of the lymphomas usually without systemic involvement at the time of the diagnosis. The incidence of C-ALCL among other types of peripheral T-cell lymphoma is 1.7\%. The peak incidence in the sixth decade of life and average age of $50 \%$ of cases are diagnosed in patients aged ${ }^{2}$ at 61 . Most patients present with solitary or localized nodules, papules or plaques but $20 \%$ patients may have multiple lesions. The lesions usually present on the trunk, extremities and buttocks. They are usually asymptomatic except mild tenderness ${ }^{3}$. Histologically, these lesions show diffuse infiltrate of anaplastic lymphocytes with characteristic morphology of oval to irregular nuclei, prominent eosinophilic nucleoli and abundant cytoplasm. The lesions usually do not present epidermotropism. C-ALCL is characterized by loss of expression of one or more T-cell antigens (i.e., CD2, CD3 and CD5) and mostly expression of CD30+ antigen. CD30+ antigen expression in $>75 \%$ of anaplastic cells is a condition for C-ALCL diagnosis. Establishing a proper diagnosis of C-ALCL is often difficult because of its histological morphology have similarity of others diseases like lymphomatoid papulosis. This case report aimed to describe a diagnosis of a rare case of C-ALCL of a young patient and its excellent therapeutic response.

\section{Case Report}

A 30 years old soldier of Bangladesh Army was reported to SOPD, Jaber Al Ahmed Armed Forces Hospital with the complains of multiple papulo-nodular lesions in the right forearm for last 06 months. The lesions were gradually increasing in size and changing the skin color. There is a history for spontaneous regression of some of them before. The largest lesion was $4 \times 3 \mathrm{~cm}$ which was non tender. His general health condition was good. The patient was evaluated clinically by surgeon and sent to cytopathology lab for Fine needle aspiration cytology (FNAC). FNAC revealed highly cellular smear with monotonous looking atypical cells with coarse chromatin. It was suspicious for skin lymphoma but diagnosis was not conclusive and advised for biopsy.Biopsy was done and send for histopathological examination. Histopathology report displaying deep dense nodular infiltrates extending through all levels of the dermis into the subcutis. The epidermis is not involved; it is separated by the infiltrates by wide clear zone. The infiltrates involve skin adnexa and some peripheral nerves. They are composed of cohesive sheets of medium sized to large cells with irregularly shaped nuclei and multiple small inconspicuous nucleoli and clear cytoplasm. The nuclei show outline irregularities embryo shaped, whorled shaped, indented forms etc. High mitotic rate is present, e.g. atypical mitoses. Background shows plasma cells and histiocytes. Small vascular capillary proliferations are seen. Immunohistochemistry revealed that he tumour cells are positive for CD30 (more than 75\%), CD3, CD43 and Vimentin. The tumour cells were negative for pan-CK, S-100, CD68, Desmin, EMA, CD 99, LCA (CD45). CD 4 and CD 8 positive small lymphocytes were found scattered or at periphery of tumour nodules, but the large cells were negative. CD7 positive subsets of small lymphocytes were detected also in fat tissue. Final diagnosis was Primary cutaneous anaplastic large cell lymphoma. The case referred to Kuwait cancer care centre (KCCC) for review and further treatment. In KCCC, besides routine immunohistochemistry, they did ALK and it was negative. So their final diagnosis was ALK -negative Primary cutaneous anaplastic large cell lymphoma. The differential diagnosis came to our consideration were - primary or metastatic skin neoplasm, Lymphomatoid papulosis and mycosis fungoides. Lymphomatoid papulosis is a chronic recurrent lymphoproliferative skin disease with self-regressing papulo

1. Lt Col Md Iqbal Karim, MBBS, MCPS, FCPS (Histopathology), Classified Specialist in Pathology, Armed Forces Hospital, Kuwait (E-mail: iqbalhistopath@gmail.com 2. Lt Col Moyassaque Ahmed, MBBS, DDV, MCPS, FCPS (Dermatology) Classified Specialist in Dermatology, Armed Forces Hospital, Kuwait 3. Maj Rajib Kumar Malakar, MBBS, Graded Specialist in Surgery, Armed Forces Hospital, Kuwait 4. Dr Rosen Nachev, MBBS, MD, Histopathologist, Armed Forces Hospital, Kuwait. 
nodular skin lesions and atypical lymphoid cells infiltrations in wedge shaped pattern in a polymorphous inflammatory background but this case absence of epidermotrophism with absence of polymorphous inflammatory cell infiltration except scattered eosinophils. Clinically patient have papulonodular localized lesion without skin ulceration or gangrene. We excluded this diagnosis of Lymphomatoid papulosis. Mycosis fungoides (MF) usually displays epidermo-tropism or lymphocytes placed into dermo-epidermal junction in vertical growth pattern. IHC MF may have the different phenotype such as: $C D 3+C D 4+C D 8+, C D 3+$ CD4+ CD 8-, CD3+ CD4+ CD56+ but in all stages CD30+ positive. In our case morphology and spreading of neoplastic infiltrate did not match differential diagnosis with MF. We excluded metastatic skin lesion as there was no nodal or systemic involvement clinically and radiologically. The patient was treated in $\mathrm{KCCC}$ with low dose methotrexate and follow up. After 03 months of treatment, all papulo-nodular lesions were disappeared and patient clinically completely cured without any residual lesion.
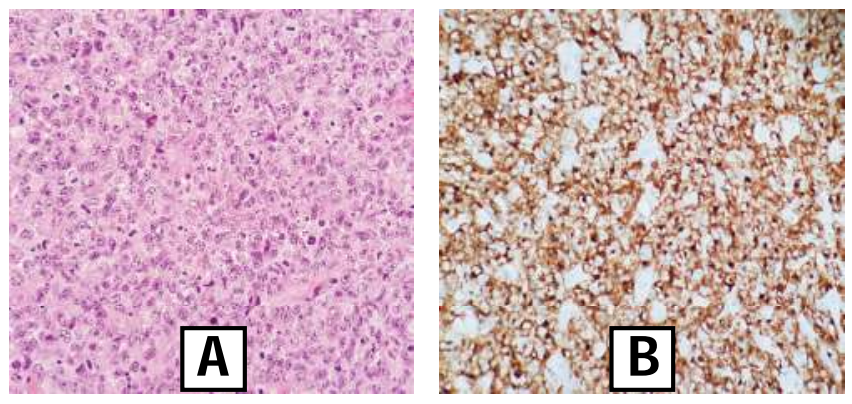

Figure-1: Cutaneous Anaplastic large Cell Lymphoma. A. Hematoxylin \& Eosin stain (H\&E) B. Immunohistochemistry shows CD 30+ cells positive cells

\section{Discussion}

Primary cutaneous anaplastic large cell lymphomas refer to clinically and biologically heterogenous group of non-Hodgkin's lymphomas (NHL) defined by clonal proliferation of cutaneous $\mathrm{T}$ lymphocytes. C-ALCL accounts for approximately $9 \%$ of all cutaneous T-cell lymphomas ${ }^{3}$. The criteria for the diagnosis of C-ALCL includes - more than $75 \%$ of CD30 + large anaplastic cells in skin biopsy and absence of clinical history of lymphomatoid papulosis, mycosis fungoides or other cutaneous lymphomas along with absence of extracutaneous localization after extensive investigations at presentation 4 . C-ALCL is also called regressing atypical histiocytosis. This is the second most common type of cutaneous T cell lymphoma. Median age 55 years, M:F ratio 2:1, occasionally children. Common form of cutaneous $\mathrm{T}$ cell lymphoma in $\mathrm{HIV}+$ individuals ${ }^{6}$. The most common locations are the scalp and extremities ${ }^{7}$. Most patients with C-ALCL present with localized solitary lesions and about $20 \%$ of patients have multiple lesions ${ }^{8}$. C-ALCL occurs as two distinct entities- a widespread systemic disease or a localized cutaneous disease. But both types have similar histological features with cohesive sheets of large lymphoid cells with dense nodular infiltrate extending through all levels of the dermis into the subcutis. The neoplasm consists of sheets of large cells with irregular nuclei and one or multiple nucleoli with eosinophilic cytoplasm. Mitoses are frequent. Clusters of small reactive lymphocytes are found within and around the tumour. Immunohistochemistry of C-ALCL shows CD30 expressed about $75 \%$ of the anaplastic lymphoid cells with variable expression of T cell antigens (CD3, CD5 and CD7) may be found. The World Health Organization classification of hematopoietic tumors and lymphoid tissues now recognize this sub classification, dividing systemic C-ALCL into ALK-positive and ALK-negative disease. C-ALCL with ALK positive usually have excellent prognosis?.

Genetics-Clonal rearrangement of $T$ cell receptor genes is detected by Southern blot and PCR in most cases (over 90\%) of C-ALCL. The translocation $t(2 ; 5)(p 23 ; q 35)$ resulting in expression of npm-alk protein which is a characteristic feature of systemic anaplastic large cell lymophomas, is rarely if ever found in C-ALCL ${ }^{10}$. Differential diagnosis was lymphomatoid papulosis and mycosis fungoides. Lymphomatoid papulosis (LymP)-Clinically this disorder is characterized by localized or multifocal (70\%) papules and nodules up to $1-2 \mathrm{~cm}$, usually asymptomatic. Spontaneous regression of skin lesions after weeks (to months) is possible. Because the morphologic and immunophenotypic features of LyP and PC-ALCL overlap significantly and as no biomarker to date has been found to reliably distinguish these entities, it is essential to correlate the pathologic findings with the clinical history. Indeed, it is the clinical behavior of LyP, characterized by recurrent and regressing crops of papules and nodules that aids in the distinction from C-ALCL ${ }^{11}$.

Prognosis and predictive factors C-ALCL has a good prognosis with 5 year-survival rates of $90 \%$. About $40 \%$ of C-ALCL show spontaneous regression of tumour. Regional lymph nodes may be involved, but the survival rate is similar to patients with skin lesions only. Extracutaneous spread occurs in $10 \%$ of the patients, especially in those with multifocal lesions with a fatal outcome in only a minority of the patients. Spontaneous regression and age less than 60 years are associated with a better prognosis, while extracutaneous disease and higher age tend to have a worse outcome.

\section{Conclusion}

As cutaneous anaplastic large cell lymphoma has excellent prognosis irrespective with appropriate treatment. So, accurate diagnosis is very important for management of patient as well as avoiding aggressive treatment for lymphoma. Patients' need close monitoring as they have the potential risk for dissemination of extracutaneous spread. It is also necessary to differentiate C-ALCL from mycosis fungoides or other lymphomas. Misdiagnosis and inappropriate/aggressive treatment may endanger the patient's life. This case report emphasised the importance of accurate diagnosis and individualized proper treatment and got the better outcome of patient. 


\section{References}

1. Ralfkiaer $E$, Willemze R, Paulli $M$ et al. Primary cutaneous CD30-positive T-cell lymphoproliferative disorders. WHO Classification of Tumours of Haematopoietic and Lymphoid Tissues. 4 th ed. France: International Agency for Research on Cancer (IARC); 2008:300-1.

2. Diamantidis MD, Papadopoulos A, Kaiafa G et al. Differential diagnosis and treatment of primary, cutaneous, anaplastic large cell lymphoma: Not always an easy task. Int J Hematol. 2009; 90(2):226-9.

3. Diamantidis MD, Myrou AD. Perils and Pitfalls Regarding Differential Diagnosis and Treatment of Primary Cutaneous Anaplastic Large-Cell Lymphoma. Scientific World Journal. 2011; 11:1048-55.

4. Pauline GM, Chao-Lo, King-Ismael $D$ et al. Primary cutaneous CD30+anaplastic large cell lymphoma: Report of a rare case. J Dermatol Case Rep. 2008; 2:31-4.

5. Kaudewitz P, Burg G, Stein H. Ki-1(CD30) positive cutaneous anaplastic large cell lymphomas. Curr Probl Dermatol. 1990; 19:150-6.
6. Kerschmann RL, Berger TG, Weiss LM et al. Cutaneous presentations of lymphoma in human immunodeficiency virus disease. Predominance of T cell lineage. Arch Dermatol. 1995; 131:1281-8.

7. Kaudewitz $P$, Stein $H$, Dallenbach $F$ et al. Primary and secondary cutaneous Ki-1+ (CD30+) anaplastic large cell lymphomas. Morphologic, immunohistologic, and clinical-characteristics. Am J Pathol. 1989; 135(2):359-67.

8. Bekkenk MW, Geelen FA, Willemze R . Primary and secondary cutaneous $\mathrm{CD} 30(+)$ lymphoproliferative disorders: A report from the Dutch Cutaneous Lymphoma Group on the long-term follow-up data of 219 patients and guidelines for diagnosis and treatment. Blood. 2000; 95:3653-61.

9. X Xing, L Andrew. Anaplastic large cell lymphomas: ALK Positive, ALK Negative and primary cutaneous. Advances in anatomic pathology. 2015; 22(1):29-31.

10. Macgrogan G, Merlio JP. CD30-positive cutaneous large cell lymphomas. A comparative study of clinicopathologic and molecular features of 16 cases. Am J Clin Pathol. 1996; 105:440-50.

11. Brown RA, Fernandez-Pol S, Kim J. Primary cutaneous anaplastic large cell lymphoma. J Cutan Pathol. 2017; 44:570-7. 